\title{
Über den medizinischen Aufschwung in den vierziger Jahren des I9. Jahrhunderts
}

\author{
Mit einem Verzeichnis der Werke von \\ Hermann Lebert (1813-1878) ${ }^{1}$ \\ Von Edgar Goldschmid, Lausanne
}

Der Aufschwung in der theoretischen und praktischen Medizin in den vierziger Jahren des 19. Jahrhunderts läßt sich auf verschiedenen Wegen verfolgen. Abgesehen etwa von der Entfaltung der Physiologie und der deskriptiven Anatomie, ist das anscheinend plötzliche Auftreten der Histologie auffallend, der normalen wie der pathologischen, in der ersten Hälfte der vierziger Jahre. Vorläufer gibt es da wohl schon seit langem, die mit einfachen Mitteln arbeiten; aber zu größerer Bedeutung gelangt die Histologie doch erst mit der Entwicklung der pathologischen Anatomie. Schon das selbständigere Auftreten derPathologie hatte den Amerikaner Godman 1825 zur Prophezeiung gebracht ${ }^{2}$, von hier aus wäre die Revolutionierung der Medizin zu erwarten. Nun hatte Henle seit 1830 kleinere histologische Arbeiten veröffentlicht, Johannes Müllers grundlegendes Werk, das Handbuch der Physiologie, war 1833 erschienen. Henles wichtigste Untersuchungen kamen seit 1837 ans Licht; das Handbuch der Anatomie (im Soemmeringschen Werk) kam 184l. Aber gerade vorher hatte die medizinische Welt Johannes Müllers nicht zu überschätzendes Buch «Über die Struktur und den feineren Bau der Geschwülste» erhalten (1838).

Ihm folgten 1840 Henles "Pathologische Untersuchungen" und seit 1846 sein «Handbuch der rationellen Pathologie»: alles Werke, die bis heute ihre Bedeutung bewahrt haben.

Nun war in den vorhergehenden Jahren, zusammenhanglos, eine große Reihe von Untersuchungen verschiedener Forscher bekanntgeworden über Chemie und Mikroskopie der Körperflüssigkeiten, wie Harn, Eiter, Blut; die Mehrzahl zwischen 1830 und 1840. Als Spätling soll nur ein Werk noch erwähnt werden, weil es die Ehre hatte, nach dem frühen Tode des Autors von Billroth 1856 herausgegeben zu werden: die "Mikrogeologie» von Meckel ab Hemsbach - mikroskopische Untersuchungen von Konkrementen.

1 Vortrag in der Schweizerischen Gesellschaft für Geschichte der Medizin und der Naturwissenschaften, St. Gallen, 4. September 1948.

2 Freundlicher Hinweis von Herrn Prof. Shryock (Phila.). 
Als zeugnisgebend für die neue Richtung können wir im weitern drei Werke betrachten: die «Icones histologiae pathologicae» von Julius Vogel, 1843; die "Physiologie Pathologique» von Hermann Lebert, 1845; und den "Atlas der Pathologischen Anatomie... der ... Veränderungen der Organe und Gewebe»... von Gottlieb Gluge, 1850. Hierzu sei bemerkt, daß "pathologische Physiologie» und "allgemeine Pathologie» gleichbedeutend sind.

Die Vogelschen "Icones histologiae pathologicae» sind 26 Kupfertafeln in Imperialquart mit 291 Figuren. Die Lebertschen Zeichnungen finden sich auf 22 Kupfertafeln, ebenso in Imperialquart, z. T. leicht getönt - sie wurden später z. T. wieder benutzt in Leberts großem "Traité d'anatomie pathologique générale et spéciale» von 1857 bis 1861. Der Glugesche Atlas der pathologischen Anatomie, 1843 begonnen, enthält zwölf Tafeln in Royalquart mit histologischen Figuren, die 1850 auch noch selbständig erschienen, ebenfalls schwarz-weiß und getönt. Nebenher sei hier erwähnt, daß bereits 1845 das erste Lehrbuch der pathologischen Histologie ebenfalls mit ein paar Tafeln erschienen ist, von der Hand des früh verstorbenen Fr. Günsburg in Breslau, einem praktischen Arzt.

Welches von diesen Werken den größten Einfluß ausgeübt, ist schwer zu sagen. Am handlichsten waren entschieden die separat erschienenen histologischen Hefte des Glugeschen Buches - ein bei den hohen Preisen der Tafelwerke nicht zu unterschätzender Umstand. Das reichhaltigste und eindrucksvollste war aber und ist noch das Lebertsche große Werk mit seinen prachtvollen, kolorierten Tafeln, welche den ersten Versuch - in der "Physiologie Pathologique» - weit hinter sich lassen. Obschon in den großen Bibliotheken und den alten Instituten vorhanden, sind Werke und Autoren heuzutage so gut wie vergessen - trotz dem riesigen Format, das sie nicht übersehen läßt! -; und doch sind sie durchaus imstande, weitgehend unser Interesse wachzurufen und Kenntnisse zu vermitteln.

Der ganze "Aufschwung» ist nun hundert Jahre alt, und die an gewohnter Stelle gegebenen Zusammenfassungen sind oft farblos, einseitig und wenig aufschlußreich. Da ist es wohl am Platz, ein paar charakteristische Einzelheiten zur Beleuchtung dieser Zeit anzuführen.

Die Rokitanskysche Humoralpathologie war 1842 erschienen und bereits 1846 vom jungen (25jährigen!) Virchow zu Fall gebracht worden. Virchow selbst mußte aus politischen Gründen 1849 seine Prosektur in Berlin verlassen, stand aber 1856 in Konkurrenz mit Billroth für die Professur in Greifswald - dem gleichen Billroth, den wir uns nur als den 
großen Chirurgen vorzustellen pflegen. Damals waren es zwei junge pommersche Landsleute, im Beginn einer Laufbahn. Zwanzig Jahre später schreibt Billroth in Wien, einigermaßen erstaunt, "daß dieser und jener Patholog damals sozusagen ins gemachte klinische Bett fiel wie Ernst Leberecht Wagner oder Lebert", - wobei man wieder einmal daran gemahnt wird, daß anderen noch lange nicht erlaubt ist, was man selber tut. Und damals, 1856, standen immerhin schon drei Professuren für Virchow offen. Zehn Jahre vorher hatte Du Bois Reymond, überrascht oder verächtlich, einem Freund geschrieben, «für die Stelle des Prosectors an der Charité sei bei der Militärpartei Stimmung für einen Eleven, Dr. Virchow, der sich mit Mikroskopie und pathologischer Anatomie nicht ohne Erfolg abgegeben haben soll»... so weit waren die physikalischen Physiologen damals von den anatomischen und klinischen entfernt. Immerhin war $\mathrm{Du}$ Bois gerade damit beschäftigt, wie er in den gleichen Briefen erzählt, seinen Chef Johannes Müller zu «dressieren».

Die naturphilosophische Ära war zu Ende; die naturhistorische war im Aufstieg, wie es sich schön am Werdegang Schoenleins verfolgen läßt. Die pathologische Anatomie schien berufen, die Führung in der wissenschaftlichen Medizin zu übernehmen. Ein richtiges Lehrbuch war wohl noch nicht vorhanden - Vetter mit seinen ausgezeichneten, doch leider unvollendeten "Aphorismen" von 1803 war so unbekannt geblieben, daß er sogar von Billroth bei der Aufzählung der österreichischen Pathologen unerwähnt bleibt. Das große Werk von Cruveilhier war alsbald nach seiner Vollendung 1842 ins Italienische und teilweise ins Deutsche übertragen worden; und kurz darauf wurde der ähnlich eingerichtete große Atlas der pathologischen Anatomie von Jo. F. Hm. Albers herausgebracht, doch ohne einen Fortschritt zu bedeuten. Er brachte wiederum nur makroskopische Anatomie und war dem Vorgänger unterlegen. Der Glugesche Atlas begann erst zu erscheinen.

Die rnikroskopische Forschung war noch im Beginn, auf Spezialitäten beschränkt, soweit sie sich nicht ausschließlich auf Botanik und Zoologie bezog. Parasitologie war im Werden; auch einige Bakteriologie war bereits vorhanden - die Grundlage der medizinischen, besonders der klinischen Mikroskopie war gelegt, denn Untersuchungen über die «Körperflüssigkeiten»(Harn, Blut, Eiter, Lymphe, Sputum) waren, wie erwähnt, in größerer Anzahl erschienen, so auch die von Jul.Vogel, die heute noch als "Anleitung zur Harnanalyse» von Vogel-Neubauer existieren.

In der eigentlichen Histologie erschien 1831 das Handbuch der Anatomie 
mit Hinweis auf die "Icones anatomicae», die C. J. Mt. Langenbeck seit 1827 herausbrachte - die bekannten acht Hefte im Imperialfolio. 1838 kam das erwähnte epochale Werk von Johannes Müller, «Über den feineren Bau der Geschwülste und ihre Formen», 1839 ließ Rudolf Wagner die «Erläuterungstafeln zur Physiologie und Entwicklungsgeschichte» erscheinen, die "Icones physiologicae». Die Orientierung unter allen diesen Büchern wird durch die damals übliche doppelte und dreifache Titelform erschwert; aber da ist daran zu erinnern, daß erst Schoenlein die lateinische Sprache in seiner Klinik zugunsten der deutschen aufgab, während die lateinische Klinik bei $W_{\text {olff }}^{3}$ weiterbestand, und daß noch der ältere Quincke $^{4}$ im Approbationsexamen lateinisch prüfte.

Nach Wagner folgten 1841 die "Beiträge zur Kenntnis der Säfte und Exkrete des menschlichen Körpers", von Julius Vogel; und vom gleichen Autor schon 1843 die "Icones histologiae pathologicae» als «Erläuterungstafeln» zu seiner pathologischen Histologie mit Rücksicht auf das «Handbuch der pathologischen Anatomie». Diese Vogelschen Tafeln sind heute noch durchaus beachtenswert und dürften sich auch in den älteren pathologischen Instituten noch überall vorfinden. Die 291 Figuren auf 26 z. T. kolorierten Tafeln (in Kupferstich) sind inhaltlich etwa das, was heute noch bei der Untersuchung frischen, pathologisch-anatomischen Materials gefunden werden kann. 1845 erschien dann, in Imperialquart, die "Physiologie Pathologique, recherches cliniques, expérimentales, microscopiques sur l'inflammation, la tuberculose, les tumeurs, la formation du cal, etc.» par le Dr. Lebert ..., médecin à Lavey. Es sind das histologische Abbildungen auf 22 Kupfertafeln, teilweise getönt; auch heute noch durchaus brauchbar. Der gleichzeitige "Cours de Microscopie au microscope daguerréotype» mit dem "Atlas du Cours» usw. von A.Donné und Foucault hat auffallenderweise kaum Spuren hinterlassen - trotz der anerkannten Verdienste des Autors, dem wir die erste Beschreibung der Blutplättchen und des Trichomonas vaginalis verdanken.

Den Abschluß dieser Reihe bilden, wie schon erwähnt, zwei große Atlanten der pathologischen Anatomie: der von Gottlieb Gluge, 1843

3 Eduard Wolff, Generalarzt und Professor in Berlin (1794-1878), leitete bis 1857 die Medizinische Klinik für Wundärzte in der Charité sowie die Medizinisch-Chirurgische Militärakademie.

${ }^{4}$ Hermann Quincke senior, Frankfurt a.d. O. und Berlin. Gesuchter praktischer Arzt und Mitglied der ärztlichen Prüfungskommission (1808-1891). Abteilungsleiter an der Charité. 
bis 1850 erschienen, mit 103 Tafeln in Royalquart, von denen zwölf Tafeln einen Atlas der pathologischen Histologie bilden. Dann der große vierbändige "Traité d'anatomie pathologique générale et spéciale», in Royalfolio, von dem schon genannten Hermann Lebert, allwo die Histologie neben der makroskopischen Anatomie zum erstenmal gleichberechtigt erscheint. Die erwähnten Autoren sind heute vergessen, obwohl die Biographien ihr Lob verkünden. Nur Langenbecks Name klingt vertraut; aber er wird zumeist mit dem jüngeren Langenbeck verwechselt, Bernhard, dem großen Chirurgen. Wer sind nun Gluge, Vogel und Lebert?

Der älteste von ihnen, Gottlieb Gluge, war 1812 in Brakel in Westfalen geboren, studierte in Berlin seit 1831 und doktorierte in Berlin 1835 mit einer pathologisch-histologischen Arbeit, den «Observationes nonnullae microscopicae fila (quae primitiva dicuntur) in inflammatione spectantes, cum tabulis». Aber schon 1833 hatte die Berliner Fakultät eine Arbeit Gluges preisgekrönt, "Die Influenza oder Grippe, nach den Quellen historisch-pathologisch dargestellt». Er ließ sich als praktischer Arzt in Minden (Westfalen) nieder und ging auf Reisen, so 1836 nach Paris. Schon 1838 wurde er Professor der Physiologie und der Medizinischen Klinik in Brüssel. Nebenher ging dort eine ausgedehnte Privatpraxis. Von seinen Arbeiten seien die "Anatomisch-mikroskopischen Untersuchungen zur allgemeinen und speciellen Pathologie» erwähnt, mit Lithographien, Minden 1839 und Brüssel 1841, die "Abhandlungen zur Physiologie und Pathologie, Anatomisch-mikroskopische Untersuchungen", Jena 1841, und der bereits genannte "Atlas der Pathologischen Anatomie oder bildliche Darstellung und Erläuterung der vorzüglichen krankhaften Veränderungen der Organe und Gewebe des menschlichen Körpers», mit 103 kolorierten Tafeln in Folio, 1843-1850. Es war deutlich ein billigeres Gegenstück zu Cruveilhier, aber mit Hinzunahme der Histologie (siehe oben) auf zwölf Kupfertafeln. Die hier beschriebenen Fettkörnchenkugeln müssen immerhin noch heute als "Glugesche Körperchen" von allen Histologiebeflissenen gelernt werden. Bei der Gelegenheit sei daran erinnert, daß die achromatischen Linsen 1830 vom älteren Lister, die Kapillarinjektion mit Karmin 1847 von Joseph Gerlach eingeführt wurde - die eigentliche Karminfärbung, die noch heute ihren Wert hat, erst 1855. Wieso dieser praktische Arzt aus Westfalen nach Brüssel ging und dort so früh eine Professur erhielt, entzieht sich meiner Kenntnis.

Hier sei nebenher ein weiterer, ungarisch-französischer Histolog er- 
wähnt, David Gruby, der in Großwardein (Ungarn) 1814 geboren, in Wien studierte und sich hauptsächlich mit Anatomie und Chirurgie befaßte. Er veröffentlichte 1839 «Observationes microscopicae, ad morphologiam pathologicam spectantes» mit 4 Kupfertafeln, und 1840 «Morphologia fluidorum pathologicarum» mit 7 bzw. 5 Tafeln. Da er in Wien nicht vorankam, ging er nach Paris, wo er bis 1898 praktizierend lebte. Auch er brachte Neues: er hielt Kurse ab über mikroskopische Anatomie und Chemie, die sich in Paris großen Zuspruches erfreuten. Auch Donné gab Kurse, doch ist mir nichts Näheres bekannt.

Im gleichen Jahr 1814 wie Gruby wurde Julius Vogel in Wunsiedel geboren, studierte in München und wurde dort 1838 mit einer Arbeit "Prodromus disquisitionis sputorum etc. continens sputorum elementa chemica et microscopica» promoviert. Dann kamen «Physiologisch-pathologische Untersuchungen über Eiter, Eiterung etc.», "Beiträge zur Kenntnis der Säfte und Excrete des menschlichen Körpers etc.» von 1841. Unterdessen war die Habilitation 1840 in Göttingen erfolgt, Vogel wurde Mitdirektor des Rudolf Wagnerschen Physiologischen Institutes und wurde 1846 als Professor nach Gießen berufen. 1843 erschienen die o. e. «Icones histologiae pathologicae» (Erläuterungstafeln) und die "Pathologische Anatomie des menschlichen Körpers» 1845. Im nächsten Jahr «Die Gesetze, nach welchen die Mischung von Flüssigkeiten und ihr Eindringen in permeable Substanzen erfolgt». Die Berufung als Professor und Klinikdirektor nach Halle erfolgte 1856. Er blieb dort bis zu seinem Tode 1880, nachdem er schließlich nur noch die Professur für Allgemeine Pathologie und Pathologische Anatomie beibehalten hatte - hochgeehrt und als einer der berühmtesten Mediziner Deutschlands, besonders als "hervorragender Patholog» bezeichnet.

Heutzutage ist kein Porträt nachzuweisen, von seinen so gepriesenen Werken sind wohl nur die "Icones histologiae pathologicae» in den älteren Instituten auffindbar - in großen Bibliotheken vielleicht eine der siebzehn Auflagen einer populären Schrift über «Die Corpulenz», die zwischen 1865 und 1879 erschienen sind. Seine gerühmte "Pathologische Anatomie» im Soemmeringschen Handbuch teilt das Los aller Handbücher; doch wird sein Anteil am Virchowschen Handbuch von 1854, "Die Störungen der Blutmischung», wenigstens als sein bedeutendstes Werk bezeichnet.

War der Lebenslauf von Julius Vogel so einfach wie glücklich, und der von Gottlieb Gluge verständlich, wenn auch nicht ganz erklärt, so ist das Leben von Hermann Lebert im Vergleich mit heutigen Verhältnissen schon 
fast märchenhaft zu nennen. Da er uns eine kleine Autobiographie hinterlassen hat, so sind wir über die Tatsachen seines Lebens, mit Ausnahme der letzten zehn Jahre, orientiert.

Der Berliner Hermann Lebert wurde 1813 in Breslau geboren und starb 1878 in Bex im Waadtland. Seit 1831 in Berlin, in Zürich und Paris mit dem Studium von Medizin und Naturwissenschaften befaßt, ließ er sich 1836 als Arzt in Bex (Waadt) nieder und wurde 1838 als Badearzt in Lavey angestellt - es ist das die Gegend, in der A. v. Haller 1758 bis 1764 als Salzdirektor von Roche und Bex lebte. Im Winter in Paris, überließ er sich von 1842 bis 1845 experimenteller und pathologisch-anatomischer Forschung. Von der französischen Regierung mit Chs. Robin zu einer zoologischen Expedition an die Nordseeküste berufen, sammelte er dort und auf den Kanalinseln Seetiere zu Musealzwecken. 1846 unternahm er zoologische Exkursionen in der Schweiz, arbeitete über Entwicklung der Fische und siedelte als praktischer Arzt nach Paris über. Von dort wurde er 1852 als Professor der Medizinischen Klinik und Krankenhausdirektor nach Zürich berufen, und 1859 in der gleichen Eigenschaft nach Breslau. 1874 zog er sich nach Vevey zurück, bis zu seinem Tod literarisch tätig. Schon früh wurde er durch Verleihung der Ehrenlegion, der Großen Goldenen Medaille für Kunst und Wissenschaft ausgezeichnet, erhielt später den Roten-Adler-Orden und war schließlich korrespondierendes und Ehrenmitglied von über vierzig wissenschaftlichen Gesellschaften und Akademien. Soviel findet sich etwa im Konversationslexikon... aus der Erinnerung und den Lehrbüchern ist er beinahe verschwunden. Sehen wir also etwas näher zu.

Das Literaturverzeichnis gibt uns von der Dissertation an 106 Nummern, ohne komplett zu sein, von der vierbändigen Ausgabe in Royalfolio mit farbigem Kupferstich und dem Handbuch bis zur balneologischen Broschüre. Seit der Promotion in Zürich 1834, mit der Dissertation «De gentianis in Helvetia sponte nascentibus» ist der rastlose Arbeiter nicht zur Ruhe gekommen. Er ging aus von seinen Zürcher Lehrern Oken und Schoenlein und Dupuytren und Louis ${ }^{5}$ in Paris. In Bex stand er in nahen Beziehungen zu Jean de Charpentier ${ }^{6}$, Oswald Heer ${ }^{7}$ und J.-J.Tschudy,

5 Dupuytren, Louis und Guersant aîné sind wohl noch überall bekannt.

6 Johann G.F.von Charpentier (Freiberg 1786-1855 Bex), der Erforscher der erratischen Blöcke, Professor der Geologie in Lausanne.

7 Oswald Heer (Niederuzwil 1809-1883 Lausanne), Professor der Botanik in Zürich, Antorität für Paläobotanik. 
in Genf mit J.-L.Prévost ${ }^{9}$, eine illustre Gesellschaft, wie sich später erweisen sollte. 1838 hat er seine mikroskopischen Studien angefangen. 1841 nahm er, der Badearzt, eine Amputatio cervicis vor, zu der er, im Winter, drei Stunden durch den Schnee nach Monthey reiten mußte. Zur Nachbehandlung ließ er allerdings einen Kollegen zurück.

In Paris gab's dann gemeinsame Arbeit mit Broca ${ }^{10}$, Robin ${ }^{11}$, Follin ${ }^{12}$, Leudet $^{13}$, und Verneuil ${ }^{14}$. Das Ergebnis war im Frühjahr 1845 etwas ungewöhnliches: die "Physiologie Pathologique», in zwei Bänden mit dem Atlas großen Formates von 22 Tafeln.

In Lavey hatte er 1844 nach der Bataille du Trient Schußwunden zu behandeln, mit Chs. de Montet ${ }^{15}$ und Henri Curchod ${ }^{16}$. Dann kam die zoologische Expedition mit Robin für französische Musealzwecke. Vergleichend anatomische und paläontologische Studien führten auch zu einer Sammlung von Versteinerungen für Schoenlein, zu dem er Ende 1845, nach Berlin, zurückging.

In Berlin kam Lebert in Beziehung zu Johannes Müller, Moritz Romberg, Virchow, Dieffenbach und Alexander von Humboldt - die «Physiologie Pathologique», sein erstes größeres Werk, ist Humboldt gewidmet.

8 Joh.Jac.v.Tschudy (Glarus 1818-1889 Jacobshof), Forschungsreisender, Zoolog und Philolog.

9 Jean-Louis Prévost (1790 Genf-1850), bekannt durch seine Arbeiten über das Spermatozoon, die Entdeckung der Froscheifurchung und die Begründung der ersten Poliklinik.

10 Über Paul Broca (1824-1880) bemerkt J.L.Pagel, er wäre «einer der befähigtesten Schüler Leberts gewesen, als Evangelist der von diesem aus Deutschland nach Paris verpflanzten pathologisch-histologischen Forschung». Sein Werk umfaßt Pathologie, Histologie und Medizingeschichte. Er hat das Sprachzentrum entdeckt und gilt als der Begründer der modernen Hirnchirurgie und französischen Anthropologie.

11 Charles-Philippe Robin (1821-1885), der vielzitierte Autor in Allgemeiner Anatomie und Histologie, Lexikograph (Nysten-Littré), Erfinder des Namens Leukocyt wie Entdecker des Ö̈dium albicans.

12 Frç.-Anthime-Eug. Follin (1823-1867), Chirurg, Ophthalmolog und Histopatholog in Paris.

13 Théodore-Emile Leudet (1825-1887) in Rouen, bekannt als hervorragender innerer Kliniker.

14 Aristide-Aug.-Stanislas Verneuil (1823-1895), berühmter Chirurgieprofessor und Begründer einer Schule, ebenso auch der «Revue de chirurgie».

15 Charles de Monthet (1817 Vevey -1888), praktischer Arzt und Chirurg, hat die erste Ovariotomie im Waadtland vorgenommen.

16 Henri Curchod (1820-1873), praktischer Arzt in Bex, hilfreich bei den französischen Publikationen Leberts. Mitglied des Conseil de santé. 
Bei Dieffenbach hat er sogar operiert, bei ihm und mit ihm. Wir heutigen fragen uns, was ist da passiert oder wie war das möglich? Ein Zoologe, ein pathologischer Anatom geht operieren; dazu mit der Ausbildung eines Studenten! Zwei Jahre zuvor hatte Oliver Wendell Holmes bereits die Ursache des Kindbettfiebers entdeckt und sozusagen unter Ausschluß der Öffentlichkeit publiziert; und die zunächst ebenso erfolglose Semmelweissche Mitteilung kam erst 1861 in Druck. Für Leberts klinischen Scharfblick spricht die Angabe Schürers, daß im Jahr 1859 «der Breslauer Professor der Geburtshilfe Lebert" der Semmelweisschen Entdeckung freundlich gegenüberstand.

Von Berlin ging Lebert zurück nach Lavey und bemühte sich dort u. a. — dem bäuerlichen Widerstand gegenüber erfolglos - um die Hebung der Fischzucht und amtete noch als Militärarzt in Thun, wobei er die Truppenmärsche als Abwechselung gewährend rühmt. 1846 kam die Übersiedelung nach Paris, und dort wurde auf Anregung von Guersant in sieben Wochen ein Manuskript von 644 Seiten mit einem schönen kolorierten Atlas fertiggestellt: die Preisschrift "Sur les maladies scrofuleuses et tuberculeuses» (von 1847, aber erst 1849 publiziert). Nachgerechnet ergibt das pro Tag eine Leistung von dreizehn Seiten Text, die mühselige Arbeit für den Atlas nicht mitgerechnet! Dieser Erfolg gegen sieben Bewerber veranlaßte den Minister, ihm das ärztliche Patent zur Praxis in ganz Frankreich ohne Examen zuzustellen. Während der Februarrevolution und der Junikämpfe war er als Chirurg tätig. Dann kam 1849 für die "Physiologie Pathologique» die Anerkennung in Gestalt des MonthyonPreises. Während der Cholera-Epidemie behandelte er in der Stadt, während viele Ärzte geflohen waren. Hier sei aber daran erinnert, daß in früherer Zeit diese Behandlung sowieso Sache des Chirurgus war, von der der Arzt sich fernhielt. Nunmehr erhielt er das Ritterkreuz der Ehrenlegion. Im nächsten Jahre wurde seine Krebsarbeit, «Traité pratique des maladies cancéreuses», von der Académie preisgekrönt.

Die Überlegung, ob die ausgedehnte Praxis, deren Ergebnis seine wissenschaftliche Arbeit erst ermöglichte, mit dem auftreibenden Großstadtleben noch zu vereinbaren wäre, wurde dadurch entschieden, daß ihm 1852 die Universität Zürich (auf Empfehlung von Escher, wie ich von Herrn Dr. Milt erfahre) die klinische Professur und Krankenhausdirektion anbot. Er heiratete noch schnell in Bex Mlle Cécile Fayod und begann 1853, vierzig Jahre alt, seine klinische Tätigkeit an der Stelle, wo er studiert und sein Lehrer Schoenlein gewirkt hatte. 
Schon im nächsten Jahr begann der große «Traité d'Anatomie Pathologique générale et spéciale» zu erscheinen (1861 vollendet), vier starke Bände im Großformat mit 200 kolorierten Stahlstichtafeln, makroskopisch und mikroskopisch, das Ergebnis 25jähriger Forschung und Sammlung. Das 1863 preisgekrönte Werk wurde alsbald im allgemeinen Teil ins Italienische übersetzt. (Im gleichen Jahr wurde er zum Offizier der Ehrenlegion ernannt.) Neben unzähligen anderen Einzelheiten findet sich hier auch (auf Tafel II, Nr. 16, des I. Atlasbandes) die erste Abbildung des Aktinomyces. Dazu gehört eine genaue chemisch-mikroskopische Beschreibung in 17 Zeilen im I. Textband. Der Fall war Lebert 1848 von Louis überwiesen worden.

Das war aber nicht alles, denn zwischendurch erschienen eine ganze Anzahl Arbeiten völlig moderner Richtung, in deutschen und französischen Zeitschriften, klinisch, anatomisch und auch klinisch-statistisch. Wir nennen nur die über Uterusmyom, Neurom, Dermoïd, Gallertkarzinom, Isthmusstenose, maligne Gelbsucht, Zusammenhang zwischen Otitis und Hirnabszeß, Cholera und Typhus. Und das alles auf Grund eines großen Tatsachenbestandes aus eigenen Fällen, wie immer getreulich angegeben wird. Trotz Klinik, Sektionssaal und Praxis gab es weiter noch naturwissenschaftliche, z. T. experimentelle Arbeiten, so über Pilzkrankheiten der Fliegen und Seidenraupen, über den Kretinismus in der Waadt. Das letzte Zürcher Werk war das «Handbuch der Praktischen Medizin» von 1859, in zwei Bänden, die drei Auflagen sahen und ins Holländische und Russische übersetzt wurden, obschon gleichzeitig noch ähnliche Werke von anderer Seite erschienen.

Die Universität Breslau berief Lebert 1859, auf Empfehlung von Frerichs, als inneren Kliniker und Krankenhausdirektor. Dazu erwartete ihn dort auch noch eine ausgedehnte Privatpraxis. Aber das alles hat seine Produktion nicht gehindert; denn schon 1860 erschien der "Gelenkrheumatismus mit Herz- und Nervenkomplikationen»; dann die «Krankheiten der Schilddrüse» die «Keratose und Ichthyose» - zwei Arbeiten, die er als besonders mühevoll bezeichnet —, die "Allgemeine Pathologie und Therapie» und das Studentenbuch der «Grundzüge der ärztlichen Praxis». Es folgten noch die zwei Bände der «Klinik der Brustkrankheiten» (1873/74), "die Krankheiten des Magens» (1878), und erst nach seinem Tode der "Traité clinique et pratique de la phthisie pulmonaire» (1879). Weiter finden sich noch viele völlig modern anmutende Arbeiten experimentellen, pathologisch-anatomischen und balneologischen Inhalts. 
Von den letzteren sei nur einer herausgegriffen, der Beitrag zum Buch über Montreux, gemeinsam mit Rambert, Ch.Dufour, F.-A. Forel und Chavannes von 1877 (das sogar Illustrationen, u. a. von G. Doré und Bocion aufweist).

Unter den historischen Arbeiten erwähnen wir die Gratulationsschrift zur 500-Jahr-Feier der Wiener Universität (1865) «Über den Einfluß der Wiener medizinischen Schule des 18. Jahrhunderts auf den positiven Fortschritt in der Medizin» und die Nekrologe auf J.-L.Prévost und Charpentier, ferner "Geßner als Arzt». Endlich die kleine Selbstbiographie von 1869.

Am 1. August 1878 ist Hermann Lebert in Bex gestorben, und damit war ein ungewöhnlicher und erfolgreicher Lebenslauf zu Ende-Naturforscher und praktischer Arzt, pathologischer Anatom und frühzeitiger Histolog, Chirurg und Krankenhausdirektor, Militärarzt und innerer Kliniker; als solcher, aus der freien Praxis heraus Nachfolger von Schoenlein und Frerichs. Aber, so groß seine direkte und literarische Einwirkung auf seine Zeitgenossen gewesen ist, eine Schule hat er nicht begründet. Immerhin hat die französische Klinik ihn zu den ihren gezählt, und zwar, wie Pagel betont, zu ihren hervorragendsten Vertretern.

Persönliche Erinnerungen an Lebert sind spärlich, ebenso wie an die anderen hier erwähnten Forscher, die ja auch alle zu hohen Jahren und Ehren gekommen sind. Heinrich Quincke kannte Lebert aus der späteren Zeit und war des Lobes voll. Geradeso äußerte sich eine Breslauer Patientin noch vierzig Jahre nach seinem Tod, über die Vortrefflichkeit seiner ärztlichen Fürsorge. Aus der Zürcher Zeit ist mir Persönliches bekanntgeworden aus den Briefen, die ein Lebertscher Assistent, der ältere Wegelin, nach Hause geschrieben hat, und deren Kenntnis ich seinem Sohn, meinem verehrten Freund Herrn Professor Carl Wegelin, verdanke. Lebert überließ demnach, in seiner Zürcher Zeit, die Klinik im wesentlichen seinem Assistenten (es gab nur einen), ein Zustand, der diesen ehrt, aber auch mit einem Übermaß von Arbeit am Krankenbett, am Schreibtisch und im Seziersaal belastete. Für die klinische Vorlesung ließ er sich vorher vom Assistenten orientieren. Dem Durchschnittsassistenten gegenüber scheint er nicht viel Interesse aufgebracht zu haben; wohl aber denen, die er schätzte. Es ist eben nicht so leicht, sich aus der freien Praxis in die festen Formen einer Universitätsklinik zu finden. Daher ist es auch begreiflich, daß sich die Zürcher Klinik bei Leberts Abgang in einem nicht gerade vorbildlichen Zustand befunden hätte. Auch nur mit dieser Klinik- 
Fremdheit läßt sich die sonst unverständliche Tatsache erklären, daß er 1855, während der Zürcher Cholerazeit, abwesend war und lange Zeit unerreichbar blieb. Denn schließlich ist doch bekannt (s. o.), daß er freiwillig während der Walliser Unruhen, während der Februar-Revolution in Paris, auf der Straße und im Lazarett gearbeitet, und während der Pariser Cholera-Epidemie die Kranken behandelt und nicht etwa die Stadt verlassen hat. Auch noch später wurde er wegen seiner Lazarett-Tätigkeit in den preußischen «Feldzügen» ausgezeichnet.

Wenn man nun Leberts literarische Tätigkeit (von 1834 bis 1878) erwägt, seine Reisen, Umsiedelungen und Expeditionen - eine von Schoenlein gewünschte Expedition nach Mosambik blieb unausgeführt -, so geht es einem etwa wie bei Ludwig Choulant ${ }^{17}$ : man fragt sich unwillkürlich, wann der Autor wohl Zeit gehabt hat, Klinik zu halten oder einen Patienten zu behandeln. Der Vergleich mit seinem Lehrer Schoenlein liegt nah - der hat nur praktiziert und Klinik gehalten. Und von ihm haben wir nur drei Publikationen: die wichtige Entdeckung des FavusErregers zählt genau zwanzig Zeilen. Aber er hat die Klinik revolutioniert und die wichtigste Schule hinterlassen.

Von den Lebertschen Werken finden sich seltsamerweise die kleinen balneologischen Schriften (s. o.) noch heute überall erwähnt. Die beiden pathologisch-anatomischen Tafelwerke sind noch völlig lebendig, wenn auch offenbar in den hundert Jahren ihrer Existenz im Unterricht so aufgebraucht, daß sie kaum je im Antiquariat auftauchen. Ebenso selten sind Vogel und Gruby. Wenn wir also daran erinnern, daß die «Physiologie Pathologique» und die Vogelschen Tafeln gleichzeitig sind, daß Gluges Werk zwischen ihnen und dem Lebertschen Atlas steht, dann ist die Entwicklung dieser ganzen pathologischen Histologie in etwa dreißig Jahren umschrieben, mit Johannes Müller und Henle als frühen Vertretern.

Als Histologen der Frühzeit haben auch Vogel, Langenbeck, Gruby große Verdienste, und Lebert und Gluge sind von besonderer Bedeutung als Vermittler zwischen deutscher und französischer Medizin. Die zwei-

17 Ludwig Choulant, in Dresden (1791-1861), Professor der theoretischen und praktischen Heilkunde, Direktor der therapeutischen Klinik, Direktor der Akademie und Medizinalreferent im Ministerium. Neben zahlreichen medizinischen Schriften und mehreren Lehrbüchern, wichtige medizin- und kunsthistorische Werke wie die «Geschichte und Bibliographie der anatomischen Abbildung» und die "Graphischen Inkunabeln für Naturgeschichte und Medizin». 
sprachigen Autoren betonen den Grund ihrer fremdsprachigen Publikation: sie wollen dem Land ihre Dankbarkeit bezeugen, das ihrer Arbeit jede Unterstützung und Förderung hat zuteil werden lassen. Die Rolle der Physiologie für den Aufschwung der Medizin in den vierziger Jahren des 19. Jahrhunderts ist sozusagen sprichwörtlich geworden. Aus unseren Ausführungen erhellt, daß auch mikroskopische Anatomie und Pathologie ihren Anteil redlich beigetragen haben. Um die Mitte des Jahrhunderts war die gesamte Medizin auf breitester Basis im Fortschreiten begriffen.

\section{HER M A N LEB ER T: LITERA T UR-VERZEICH IS}

Aus den Biographischen Notizen usw., Breslau, Korn, 1869. ${ }^{0}{ }^{0}$, p. $53-62$ :

Übersicht der von mir bekannt gemachten größeren Werke und sonstigen wissenschaftlichen Arbeiten

\section{Größere Werke aus dem Gebiete der praktischen Medizin, Chirurgie und pathologischen Anatomie}

1. Physiologie pathologique. 2 vols, avec un atlas de 22 pl. Paris, J. B. Baillière, 1845. Von der Pariser Akademie der Wissenschaften 1848 gekrönt.

2. Abhandlungen aus dem Gebiete der Chirurgie und der pathologischen Physiologie. Berlin, Veit 1847.

3. Traité pratique des maladies scrofuleuses et tuberculeuses. Paris, Baillière, 1849. Von der Pariser medizinischen Akademie mit dem Portalschen Preise 1847 gekrönt. Von Köhler ins Deutsche übersetzt.

4. Traité pratique des maladies cancéreuses et des affections curables, confondues avec le cancer. Paris, Baillière, 1851. Von der Pariser Akademie der Wissenschaften 1852 gekrönt. Im Auszuge ins Italienische übersetzt, in den Annali d'Omodei.

5. Anatomie pathologique générale et spéciale. 2 vols gr.-in-folio et 2 vols du même format de $200 \mathrm{pl}$. gravées en acier et coloriées. Paris, Baillière, 1857-1861. Von der Pariser Akademie der Wissenschaften 1863 gekrönt. Der allgemeine Teil ist von De la Chiaia in Neapel ins Italienische übersetzt worden.

6. Vorträge über die Cholera. Erlangen, Enke, 1854.

7. Die Cholera in der Schweiz. Frankfurt, Meidinger, 1856.

8. Handbuch der praktischen Medizin. 2 Bde. Tübingen, Laupp \& Siebeck, 1859; die 2. Auflage 1860, die 3. Auflage 1862. Ins Russische und Holländische übersetzt.

9. Über akuten Gelenkrheumatismus. Erlangen, Enke, 1860. Ins Holländische übersetzt.

10. Über die Krankheiten der Schilddrüse. Breslau, Maruschke \& Berendt, 1862. 
11. Über Keratose oder die durch Bildung von Hornsubstanz erzeugten Krankheiten. Breslau, Morgenstern, 1863.

12. Allgemeine Pathologie und Therapie, mit besonderer Rücksicht auf die praktische Medizin. Tübingen, w. o., 1864.

13. Grundzüge der ärztlichen Praxis. Tübingen, w. o., 1867 und 1868. Ins Polnische übersetzt.

II. Medizinische, chirurgische, pathologisch-physiologische und pathologisch-anatomische Abhandlungen

14.- 17. Comptes-rendus des Eaux de Lavey. Lausanne 1839, 1840, 1841 und 1842.

18.-21. Jahresberichte über die Zürcher medizinische Klinik.

Für 1853 in den Berichten des Zürcher Gesundheitsraths 1854 und in der Gazette médicale 1854 .

Für 1854 Zürich ibidem, für 1856 ibidem und beide in V.A. XII, 479, und XIII, 113 und 358.

Für 1857 in der Wiener medizinischen Wochenschrift 1858.

22. Über die Hydatiden der Leber beim Menschen. Müllers Archiv 1843.

23. De la formation du cal après les fractures. Annales de la Chirurgie française 1844.

24. Über Tuberculosis. Müllers Archiv 1844.

25. Mémoires sur les plaies par armes à feu. Arch. gén. de Méd. 1845.

26. Mémoire sur l'emploi médical du chlorure de magnésium. Arch. 1844.

27. Über die Geschwülste der Brustdrüse. Schweiz. med. Z. 1846.

28. Über die Krankheiten der Schilddrüse: Supplementarband des Berliner medizinischen Wörterbuches. Berlin 1848.

29. Expériences sur l'action de l'éther sulfurique. Gaz. méd. 1847.

30. Über die mikroskopische Diagnose des Krebses im Lebenden, vor der Operation. Roser und Wunderlichs Archiv für physiologische Heilkunde 1848.

31. Über die Vertheilung des Cretinismus im Canton Waadt in der Schweiz. Ebenda, 1848.

32. Anatomie, Pathologie und Therapie der Lymphdrüsentuberkulose. Ebenda, 1849.

33. Uber Magenkrebs. Ebenda, 1849.

34. Des maladies des os chez les scrofuleux. Mém. précédé d'un travail sur la formation des os. Mém. Ac. de Méd. de Paris 1850.

35. Un cas de pleurésie avec phénomènes amphoriques, sans tubercules. Gaz d. hôpitaux de Paris 1851. (Erster derartiger beschriebener Fall.)

36. De l'hydrocèle du cou. Mém. de la Soc. de Chir. de Paris. T. III, p. 45, 1852.

37. Du névrôme. Ibid., T. 3, p. 267, 1853.

38. Du cancroïde des diverses régions du corps. Ibid., T. 2, 1851.

39. Mémoire sur les cancroïdes cutanés. Mém. Soc. de Méd. d'observation T. 3. 1856.

40. Des changements vasculaires que provoque l'inflammation, travail précédé d'une esquisse historique sur les travaux récents sur l'inflammation. Mém.Soc, de biologie de Paris. T. 4, p. 67, 1853.

41. Des kystes dermoïdes et de l'hétérotopie plastique. Ibid., p. 203 
42. De la structure des tumeurs fibroïdes de l'utérus. Comptes-rendus des séances de la Soc. de biologie T.4, première partie, p. 68 .

Zahlr. Arbeiten in den Sitz.-Ber. d. Soc. anat. de Paris und der biol. u. chirurg. Ges., besonders von 1843-1853. Darunter eine größere:

43. De l'hypertrophie partielle de la glande mammaire. Bull. Soc. anat. 1850 .

Von wissenschaftlichen für den Moment ihrer Bekanntmachung wichtigen Berichten sind noch zu erwähnen:

44. Du pemphigus des nouveau-nés. Bull. Soc. anat. Paris. T. 26, p. 320, 1851.

45. Sur un cas d'affection syphilitique du crâne, présenté par M.Dufour. Bulletins etc., p. 141, 1851.

46. Über Gehirngeschwülste. V.A. Bd. 3, p. 463.

47. Beitrag zur Kenntnis des Gallertkrebses. V. A. IV, p. 192, 1852.

48. Über Verengerung der Aorta in der Gegend des Botallosehen Ganges. V.A. IV, p. 327, 1852.

49. Über Icterus typhoïdes. V. A. VII, p. 344, 1855.

50. Uber Entzündung der Hirnsinus infolge von innerer Ohrenentzündung. V. A. IX, 1856, p. 311 und 381.

51. Über Gehirnabscesse. V.A.X, 1856, 78, 357, 426.

52. Uber einen Fall von Ursprung der Aorta a.d. rechten Ventrikel mit Offenbleiben der Foetalöffnungen, usw. V.A. 28, p. 405, 1863.

53. Über Ursprung und Entwicklung der Doctrinen über Syphilis. Prager Vierteljahresschrift 1857.

54. Über Abortivtyphus, Prager Vierteljahresschrift 1857.

55. Pathologische Anatomie des Abdominaltyphus. Ibid. 1857.

56. Uber die in Island herrschende Hydatidenkrankheit. Monatsschrift des Zürcher wissenschaftlichen Vereins 1858.

57. Neue Beobachtungen über Dermoïdkysten. Ibid. 1858.

58. Über die gefährlichen Nervenerscheinungen beim akuten Gelenkrheumatismus. Ibid. 1860 .

59. Cas de ramollissement du cerveau par obstruction embolique de l'artère Sylvienne. Gaz. méd. de Paris 1859.

60. Ein Fall von Aneurysma dissecans der Aorta. Verhandlungen der schlesischen Gesellschaft für vaterländische Cultur 1861. Breslau 1862. (Vortrag vom 1.Dec. 1861.)

61. Über den Einfluß der Wiener medizinischen Schule des 18. Jahrhunderts auf den positiven Fortschritt in der Medizin. - Gratulationsschrift im Auftrag der Breslauer Universität zur Feier des 500jährigen Jubiläums des Wiener Universität. Berlin, Hirschwald, 1865.

62. Über das Aneurysma der Bauchaorta und ihrer Zweige. Ibid., 1866.

63. Über die Aneurysmen der Hirnarterien, eine Abhandlung in Briefen an Herrn Geh.-Obermedicinalrath Prof. Dr. Frerichs in Berlin. Berl. Klin. Wschr. 1866 und gesondert abgedruckt, Hirschwald, 1866.

64. Über die subkutane Anwendung des Morphiums als Mittel, um die Schmerzen der Geburt und der Krampfwehen zu mildern. Berl. Klin. Wschr. Nr. 11, 1866. 
65. Lettres sur la maladie provoquée par les trichines, adressées à M. le Baron Larrey. Gaz. méd. de Paris 1866 (Und separat).

66. Vorträge über Lazarettkrankenpflege. Berlin, Hirschwald, 1866.

67. Observation sur un cas de crachement sanguin particulier, d'hématinoptyse. Gaz. méd. de Paris 1866.

68. Quelques expériences sur la transmission des tubercules. Bull. de l'Ac. Impériale de méd. 15/XI 1866 (1867).

69. De l'anatomie pathologique de la pneumonie disséminée chronique, etc. Gaz. méd. 1867.

70. Über den Einfluß der Stenose des Conus arteriosus des Ostium pulmonale und der Pulmonalarterie auf die Entstehung der Tuberkulose. Berl. Klin. Wschr. 1867.

71. Beiträge zur Experimental-Pathologie der herdartigen, umschriebenen, disseminierten Lungenentzündung, sowie der Übertragung der sogenannten Tuberkulose usw. von Menschen auf Thiere. Lebert und $W y \beta$. V. A. 40, p. 142 ff. 1867.

72. Über Cavernenbildung in den Lungen nach Impfung von peritonealen Tuberkelgranulationen. Lebert und $W y \beta$. V.A.41, p. 540 ff. 1867.

73. Krankheiten der Blut- und Lymphgefäße. In Virchows Handbuch der Pathologie und der Therapie, Bd. V. Erlangen, Enke, 1867. 2. Auflage. (Die gleiche Arbeit viel kürzer, erschien in der 1. Auflage 1855.)

74. De l'empoisonnement par le phosphore. Arch. gén. de Méd. 1868.

75. Sur une petite épidémie de typhus à rechute, obs. à Breslau. Bull. de l'Ac. de méd. Sept. et oct. 1868. Paris 1868.

76. Beiträge zur Statistik und Ätiologie des Catarrhs der Athmungsorgane. Berl. Klin. Wschr. 1869.

77. Pharmacopoea Policlinici Vratislaviensis. Breslau 1869.

78. Über die Verstopfung der Bronchien durch fibrinöse Bildungen. Dtsch. Arch. klin. Med. Erlangen. 1869.

\section{Physiologische, vergleichend-anatomische und naturwissenschaftliche} Abhandlungen

79. De gentianis in Helvetia sponte nascentibus. Diss. inaug. Zürich 1834.

80.-83. Quatre mémoires sur la formation des organes de la circulation et du sang, publiés en commun avec (J.-L.) Prévost. Ann. Sc. nat., avec dessins. Paris $1844-1846$.

84. De la formation des muscles chez les animaux vertébrés, avec planches. Ibid. 1849.

85. De la structure des muscles dans les diverses classes d'animaux, avec dessins. Ibid. 1849.

86. Beobachtungen über die allgemeine Anatomie der niederen Tiere, mit Ch. Robin. Müllers Archiv 1846.

87. Über die Mundorgane der Gasteropoden, mit Abb. Ibid. 1847.

88. De la fécondation du Calmar. Observation publiée en commun avec M. Chs. Robin. Revue zool. des Bull. de la Soc. philomatique. Paris 1845.

89. Über die Pilzkrankheit der Fliegen. Mit Abb. Denkschriften der Schweiz. Naturforschenden Gesellschaft 1857. 
90. Über die Pilzkrankheit der Fliegen und die neueste in Oberitalien herrschende Krankheit der Seidenraupen mit Parasitenbildung, nebst einer Zusammenstellung der wichtigsten pflanzlich-parasitischen Krankheiten, welche für Insecten und Myriapoden bekannt sind. V. A. XII, p. 144, 1857.

91. Über die gegenwärtig herrschende Krankheit des Insects der Seide, mit Abb. Berlin 1858.

92. Über einige neue pflanzliche Parasiten lebender Insecten, mit Abb. Siebold und Köllikers Zeitschrift für Zoologie 1859.

\section{Biographische Skizzen und öffentliche Vorträge}

93. Esquisse biographique de J.L.Prévost de Genève. Gaz. méd. de Paris 1850.

94. Conrad Geßner als Arzt. Zürich 1854.

95. Nekrolog des Naturforschers Johann von Charpentier. Zürich, Verh. Naturf. Ges. Bd. IV, 1855.

96. Skizzen aus dem Leben der Seidenraupe. Abh. des Zürcher wiss. Vereins. 1857.

97. Das Engadin und seine Heilquellen. Breslau, Korn, 1869.

98. Über den Einfluß der Anatomie auf die Culturgesehichte. Breslau 1862.

\section{Nachtrag}

99. Biographische Skizzen und Überblick der von mir bekannt gemachten Werke und kleineren Arbeiten. Breslau, Korn, 1869.

100. Über Milch- und Molkenkuren und über ländliche Kurorte für unbemittelte Brustkranke. Berlin, Hirschwald, 1869.

101. Vorträge an der Breslauer Medizinischen Klinik und Poliklinik. Med. Times \& Gaz. London 1869.

102. Klinik der Brustkrankheiten. 2 Bde. Tübingen 1873/74.

103. Bex in der Schweiz, als Sommer- und Winter-Kurort. Berl. Klin. Wschr. 11, 1874.

104. Über die Indicationen für Vevey und die Bucht von Montreux als klimatische und Trauben-Kurorte. Berl. Klin. Wschr. 12 (5 Fortsetzungen), 1875.

105. Notice médicale in "Montreux», Texte par Rambert, Lebert, Dufour, Forel et Chavannes. Neuchâtel, Furrer, 1877, p. 171-199 (Bilder von Doré, Bocion).

106. Krankheiten des Magens. Ibidem 1878.

107. Kurorte der Riviera (Genua bis Nizza). Berl. Klin. Wschr. 15, 1878.

108. Traité clinique et pratique de la phthisie pulmonaire. 1879. 